\title{
First report of submandibular and parotid abscess due to Nocardia asteroides
}

\section{Authors}

Kiran Chawla

Chiranjay

Mukhopadhayay ${ }^{1}$

Prashanth V Shetty ${ }^{1}$

${ }^{1}$ Department of

Microbiology, Kasturba Medical College, Manipal, India.

\begin{abstract}
Herein, we are presenting a first case report where multiple salivary glands abscesses were diagnosed to be due to N.asteroides. Minor trauma of buccal mucosa, due to cleansing of teeth unhygienically, was most probable mode of infection. It was managed successfully by surgical drainage and post surgical co-trimoxazole therapy.
\end{abstract}

Keywords: communicable diseases, emerging bacteriology, gram-positive bacteria.

[Braz J Infect Dis 2010;14(5):544-545]@Elsevier Editora Ltda.

\section{TO THE EDITOR,}

A 32-years-old female was admitted on 12/4/10 with one month history of progressively increasing painful swelling on both sides of her face in submandibular region (Figure 1), as well as in right parotid region. There was also history of on and off fever for last one month. Earlier she visited a local doctor who treated her with analgesics and antipyretics for the swellings in the submandibular regions. The symptoms did not subside. Subsequently she noticed swelling on her right side of face in parotid region also. She was a known epileptic patient for which she took carbamazepine, and presently not on any antiepileptic treatment for the past six years. There was no past history of trauma on the face. She had the habit of cleaning her tooth-spaces with broomstick after having meals. There was no history of diabetes or hypertension. She was not on any prolonged treatment with steroids. Her general physical examination was normal. Oral examination did not show any signs of dental caries. Local examination showed:

1. A $3 \times 3 \mathrm{~cm}$ swelling, firm, mobile, tender, with erythema and local rise of temperature present in the right submandibular region.

2. A $3 \times 3 \mathrm{~cm}$ swelling in the right parotid region, firm, tender, mobile, with local temperature rise.

3. A $4 \times 4 \mathrm{~cm}$ swelling in the left submandibular region, firm, mobile, tender with local temperature rise.
Figure 1: Photograph showing right submandibular and parotid abscess by N.asteroides.

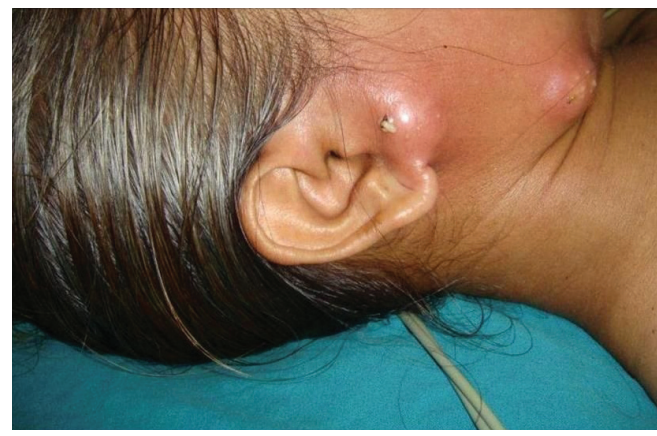

Blood sample examination showed Total Leukocyte Count (TLC) as 19,600 cells $/ \mathrm{mm}^{3}$ (Neutrophils 73.1\%, Lymphocytes 19.4\%, Monocytes 6.5\%, Eosinophils 0.7\%, Basophiles $0.3 \%$ ), Erythrocyte Sedimentation Rate (ESR) $32 \mathrm{~mm}$ at the end of first hour, Hemoglobin 11.2 gm\%, Hematocrit 34.1, platelet count $385,000 \mathrm{c} / \mathrm{mm}^{3}$, blood glucose $93 \mathrm{mg} / \mathrm{dL}$, blood urea $6 \mathrm{mg} / \mathrm{dL}$, serum creatinine $0.8 \mathrm{mg} / \mathrm{dL}$, serum sodium 140 $\mathrm{mEq} / \mathrm{L}$ and serum potassium $4.1 \mathrm{mEq} / \mathrm{L}$. Incision and drainage was done on 13/4/10. Gram staining of drained pus showed Gram positive filamentous acid fast bacilli with Kinyoun staining. Culture of pus sample grew Nocardia after 48 hours of aerobic 
incubation on sheep blood agar and nutrient agar (Figure 2). It was later speciated biochemically as N. asteroides. Antibiotic sensitivity done by Kirby-Bauer method showed sensitivity to co-trimoxazole, amikacin, cefotaxime, tetracycline and amoxicillin-clavulanic acid, but was resistant to ampicillin and erythromycin. Patient started co-trimoxazole after surgery and is on further follow-up.

Figure 2: Nutrient agar plate showing growth of Nocardia asteroides.

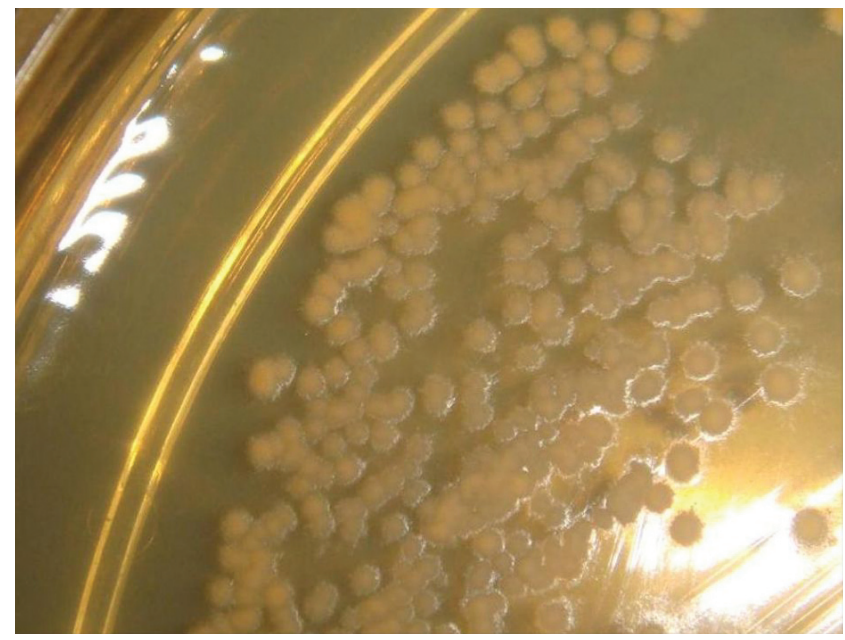

Primary cutaneous nocardiosis accounts for $5 \%$ of all nocardial infection. ${ }^{1}$ It can be divided into four clinical types: mycetoma, lymphocutaneous infection, superficial infection (abscess or cellulitis), and disseminated disease with cutaneous involvement. ${ }^{2}$ Our case is an example of primary superficial cutaneous infection. Most of the cutaneous infections are due to $N$. brasiliensis. To our knowledge only one case of submandibular abscess due to $N$. farcinica has been documented, but that patient was suffering from nephrotic syndrome and was on prolonged steroid therapy. ${ }^{2} \mathrm{~N}$. asteroides is an opportunistic pathogen, and in exceptionally rare cases it causes infection in immunocompetent persons. Usually the skin involvement by $N$. asteroides is secondary to dissemination from pulmonary disease but rarely primary cutaneous disease can be seen. ${ }^{3}$ Inoculation of Nocardia into traumatic wound is the usual mode of infection in cutaneous nocardiosis, varying from contaminated abrasions and puncture wounds to insect and animal bites. ${ }^{4}$ Cellulitis that develops in these cases is usually subacute with pain, erythema, swelling, and warmness at the affected site. If there is a delay in treatment, it may rarely disseminate to other areas involving bone, muscles and joints. ${ }^{4}$ In our case, the patient may have got the traumatic inoculation of the pathogen due to her unhygienic practice of cleaning the tooth-spaces with broomstick after every meal. Due to her unusual practice she may have got minor mucosal trauma in the past which remained unnoticed. $N$. asteroides otherwise can be present as colonizer in oral cavity and upper respiratory tract. The diagnosis of nocardiosis is usually made by microscopy and culture isolation of the pathogen. Species identification is done biochemically and more recently by molecular techniques. ${ }^{4}$ Trimethoprim-sulfamethoxazole is the drug of choice for treating Nocardia infections. For immunocompetent persons, 2-4 months treatment is given for localized infections. ${ }^{5}$

This case highlights the fact that Nocardia can be responsible for submandibular or parotid abscess, and should be considered as one of the differential diagnosis for such abscesses. It also shows the importance of keeping good oral hygiene. Correct and timely diagnosis can help us for the effective management of cases.

\section{ACKNOWLEDGEMENTS}

We acknowledge the help provided by Dr Annet D'Souza and Dr. Odaiyappan Kannappan in diagnosis and management of the case.

\section{REFERENCES}

1. Corazza M, Ligrone L, Libanore M, Virgil A. Primary cervicofacial nocardiosis due to $N$. asteroides in an adult Immunocompetent patient. Acta Derm Venereol 2002; 82:391-2.

2. Shimizu T, Furumoto H, Asagami C, Kanaya K, Mikami Y, Muto M. Disseminated subcutaneous Nocardia farcinica abscesses in a nephrotic syndrome patient. J Am Acad Dermatol 1998; 38:874-6.

3. Tsuboi R, Takamori K, Ogawa H, Mikami Y, Arai T. Lymphocutaneous nocardiosis caused by Nocardia asteroids. Arch Dermatol 1986; 122:1183-5.

4. Brown-Elliott BA, Brown JM, Conville PS, Wallace RJ. Clinical and Laboratory features of the Nocardia spp. based on current molecular taxonomy. Clin Microbiol Reviews 2006; 19:259-82.

5. Saubolle MA, Sussland D. Nocardiosis: review of clinical and laboratory experience. J Clin Microbiol 2003; 41:4497-501. 